\title{
Dynamic diagnostics of moving ferromagnetic material with the linear induction motor
}

\author{
Krzysztof Szewczyk ${ }^{1, *}$, and Tomasz Walasek ${ }^{2}$ \\ ${ }^{1}$ Częstochowa University of Technology, Faculty of Electrical Engeneering, Str. Armii Krajowej 17,42-200 Częstochowa, \\ Poland \\ ${ }^{2}$ Częstochowa University of Technology, Faculty of Mechanical Engineering and Computer Science, Str. Armii Krajowej \\ 17,42-200 Częstochowa, Poland
}

\begin{abstract}
The paper presents the application of a three-phase induction motor as a sensor measuring the force of the electromagnetic field connection between the engine and produced sheet steel. The force interaction between the engine and the manufactured sheet metal treated as a treadmill for a linear motor may be an indicator of damage to the material. Detection of places where the sheet does not meet the quality requirements may be very useful in the production process. FEM calculations were performed in the ANSYS MAXWELL environment. The results suggest the possibility of using this type of construction to test the quality of produced materials. The computational results and their analysis are presented in this article.
\end{abstract}

\section{Introduction}

The process of producing materials such as wire and sheet metal requires material control. As the product is reeled in during the final phase, a possibility to inspect the produced material disappears [1]. The top layer covers the bottom layer. Repeated control requires rewinding the material [2]. The testing method often requires cutting off a sample such as an Epstein frame.

The authors attempted to design and simulate an inspection device for the final phase of sheet metal production.

\section{The measuring principle}

The idea of measuring principle is illustrated in Figure 1. The part of the drawing on the left shows the metal sheet production area. The produced sheet metal is moved to the right in the direction of the winder.

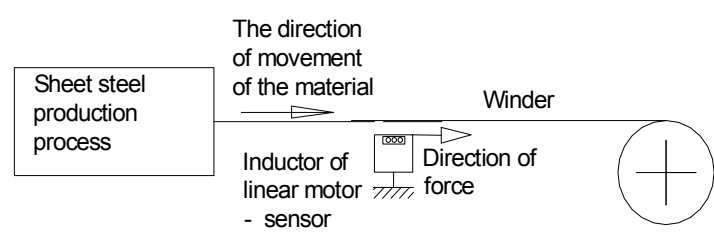

Fig. 1. Idea of diagnostic system.

A sheet metal sensor was placed before the winder. It can be connected to a device that identifies the position of the sheet. The identification device may be helpful in finding a broken piece of sheet metal later. The identified sheet is wound onto the winding shaft.

A liner electrical motor is used as a sensor. Before winding, the metal sheet passes the inductor of the electrical line motor. Depending on the direction of field movement and the direction of product movement, the sensor will show the force value. The force acting on the exciter depends on the magnetic and mechanical properties of sheet steel. With the constant sheet metal moving, any change in the magnetic properties of the sheet will change the parameters acting between the sheet and the sensor.

The main purpose of this article is to identify phenomena occurring in the sensor and around it. Special attention will be paid to the impact of an air gap as a material defect. All the calculations and their analyses are presented in the paper.

It is the authors' belief that the conducted analysis and the conclusions drawn from the project will encourage further research and contribute to the development of diagnostics of this kind.

\section{Space: 2D or 3D}

The simulation of the electromechanical system was performed in the Ansys Maxwell environment. It is an ideal environment for simulating different electromechanical converters and electromagnetic field distribution. Electrotechnical metal sheet constitutes the main part of the simulated system, being simulated as both the tested product and a sensor. Sensor construction is based on the principle of linear induction electric motor. All these elements can be simulated in the Ansys

Corresponding author: szewczyk500@gmail.com 
environment with the FEM method and the Stress Tensors Maxwell calculations. This procedure allows you to calculate the distribution of the magnetic field in the simulated system, and further to calculate the forces and torques generated by the system under test.

It is possible to analyse this kind of problems in 2D and $3 \mathrm{~d}$ in Ansys Environment. The 3D model is capable of analysing the problem without the limitation of the current flow range. In Figure 2, basic differences between $2 \mathrm{D}$ and $3 \mathrm{D}$ models have been presented. In the 2D model, on the left, the discussed currents can flow perpendicularly to the surface. Treating the computer screen as the analysed surface the currents can flow in and out in the perpendicular direction to the screen. In the 3D model we can analyse additional currents flow parallel to the screen surface. This enables analysis of boundary effects and their impact on the studied phenomena. 3D modelling of the discussed problem requires enormous computing power, large memory areas and time consuming calculations. Reducing the space of analysis to a 2D model diminishes the prospect of simulation and limits the flow of currents only in a direction perpendicular to the plane of the analysed space (Fig. 2).
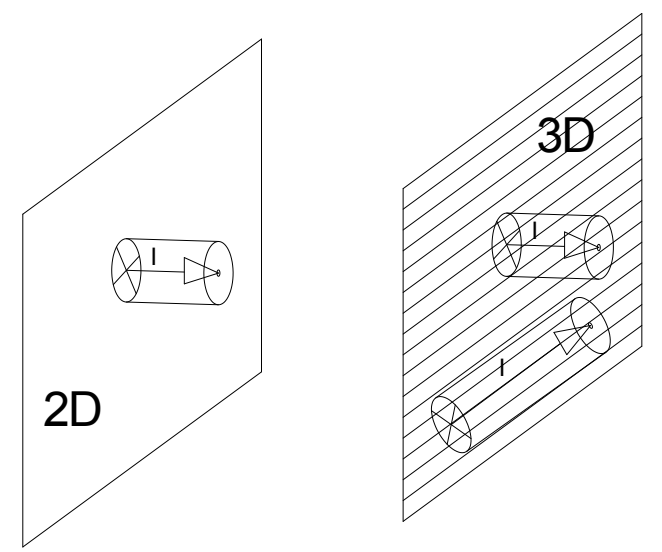

Fig. 2. Direction of currents flow in $2 \mathrm{D}$ and $3 \mathrm{D}$ simulations.

Reducing the analysed space to the two-dimensional model accelerates computations and simplifies the analysis of phenomena. However, it requires a precise design of the experiment so as not to lose the opportunity to draw the right conclusions as a consequence of limited area of analysis.

The problem discussed in the paper does not require modelling of the influence of boundary phenomena on the system. It is intended to present the idea of using a typical construction of an inductive linear motor for dynamic ferromagnetic product identification. As the boundary phenomena occurring in the construction of the linear motor drive are not the subject of this article, the 2D space was used for simulation. Presented linear phenomena in linear motors were based on common knowledge.

\section{Assumption for the Project}

The tested system consists of two basic components: the inductive linear motor and the test plate. The linear motor, shown in Fig. 3, consists of magnetic circuit and windings. The rotor of the linear motor is a sliding steel sheet. It is a treadmill for the linear engine. In a threephase motor for the field to move the engine should be composed of 15 coils. The movement of the field will generate eddy currents in the treadmill, which in turn will generate forces between the treadmill and the engine. The position of the motor working as a defect sensor for the controlled sheet is shown in Fig. 3.

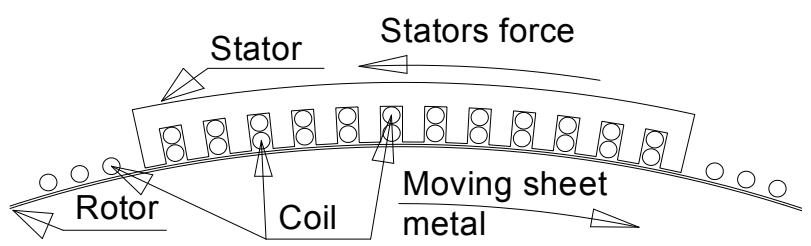

Fig. 3.Three-phase inductive linear engine. Sensor.

Winding the linear motor requires additional explanation. In the case analysed by the authors, the principle is analogous to that of an induction arc motor. In this type of engines magnetic circuit is built on the basis of an open arc rather than a closed circle shape. Due to edge effects the specific winding of the induction motor is required. Windings at the beginning (on the left) and at the end (on the right) of the magnetic circuit have coils not covering the magnetic circuit. The shape of the construction shown in Fig. 3, based on the radius, is explained in the next sections of the paper. It is related to the limited space of analysis resulting from memory constraints of the processor. The presence of additional windings outside the magnetic circuit is required by the occurrence of longitudinal boundary phenomena resulting from the limited length of the inductor of the induction motor. Longitudinal boundary phenomena consist of the formation of an alternating field (in addition to the moving component) and the input and output at the ends of the inductor, also described as marginal phenomena.

The phenomenon of induction changes B described in the function of time can be presented in the form of two components: the alternating field and the moving field as in equation (1):

$$
B=B_{t}\left(\cos \left(\omega t-\frac{\pi}{T} z\right)+B_{p} \sin \left(\omega t-\frac{\pi}{2}\right)\right.
$$

where: $B_{t}, B_{p}$ are the induction of moving and alternating field, $\mathrm{T}$ is the period of induction distribution along the axis of length $Z$ of the driver in the direction of the moving sheet. Explanation of the field compensation principle $B_{p}$ is shown in Fig. 4 . 


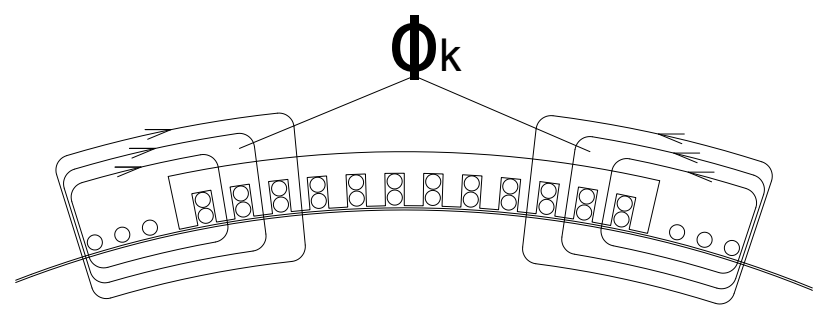

Fig. 4. Streams compensating the boundary phenomena of a linear motor.

Stream $\Phi_{k}$, consisting of two components located on the edges of a linear motor is compensated in the magnetic circuit. Compensation of the component $B_{p}$ is important because of the additional power losses it generates and the braking forces occurring in the absence of compensation. In the design of real arc-line systems, two-layer windings with the sides of the coils outside the magnetic circuit are used to compensate the boundary effect as shown in Fig. 4.

Under such assumptions, the flow of currents in individual coils was routed according to the pattern of a linear motor winding with 12 coils of the magnetic circuit (Fig. 5).

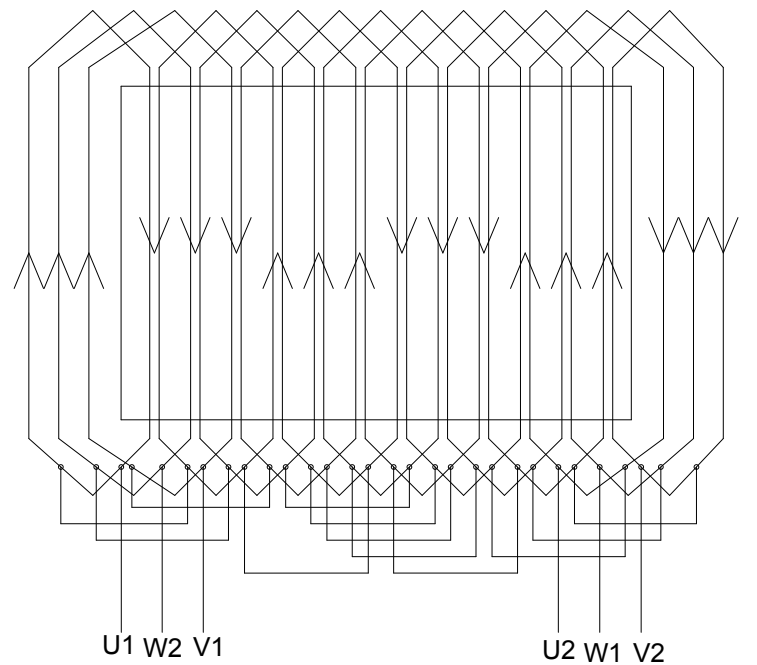

Fig. 5. Winding of the linear engine with direction of the currents flow.

Such a design of the linear motor generates a running field that interacts with the treadmill at the levelled pulsating field. The parasitic effect of the boundary effect disappears at the extension of the linear motor inductor. For relatively short structures, it should be compensated by coils located outside the magnetic circuit as proposed in the article.

\section{Simulation of infinite metal sheet length}

Simulation processes do not tolerate infinity. Computer memory, the limited area of the simulation of the phenomenon, does not allow the use of the notion of infinity, hence the produced sheet is "only" very long.
Simulation of length dimension makes it difficult to model such an object. We propose a solution to this problem: a special design of the simulated system. Figure 6 shows a proposal for the analysis of a practically infinitely long sheet. Taking into consideration the ratio of sheet width to length, e.g. $1 \mathrm{~m}$ to $10 \mathrm{~km}$, which can be easily wound on a single roll of the output product, the term infinity can be considered in the simulation of the real system. The authors propose to simulate the interaction of forces and torques of the electromechanical system as shown in Fig. 6.

The sheet is a circle with the diameter of $1 \mathrm{~m}$ and the width of $1 \mathrm{~m}$. The sensor, located at the top of the circle, is a linear motor. Because of the shape of the sheet metal, it is a section of the induction motor (arc motor). The axis of rotation is located in the centre of the sheet metal. Analysis of the forces generated by that engine is reduced to the analysis of torque versus the rotation axis of the sheet metal. The fragment of the analysed sheet contains delamination.

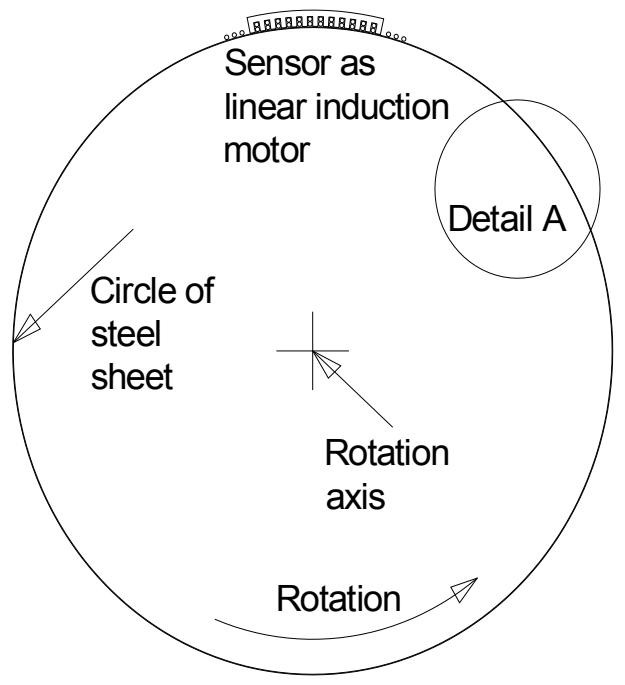

Fig. 6. Schematic process adopted for the calculation model.

The detail A shown in Fig. 7 shows the defect modelled in the article. The air gap formed during the roll of sheet metal disqualifies the product. It can be formed at the stage of the air bubble hidden in the material rolled in the production process.

Due to the place of occurrence (inside the sheet) finding the defect is impossible without special test methods and equipment (e.g. Epstein apparatus). Identifying defects requires complex tests and is not possible at the production stage. If no defect is detected, the sheet is wound on the reel drum. Finding a piece of sheet with a flaw after the end of production is practically impossible. There is a possibility to correlate the sensor system with the measurement of sheet length. This kind of electronic identification allows, at a later stage, during the product distribution, to separate the defective material. 


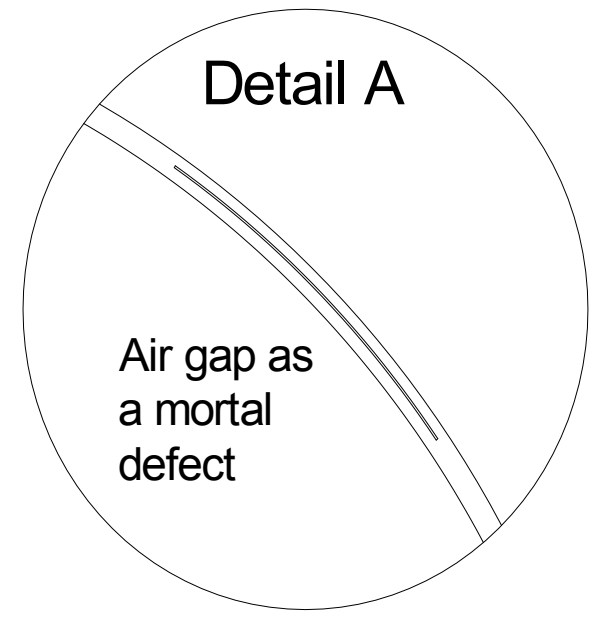

Fig. 7. Airgap. Mortal defect in produced material.

In simulations for the calculation of flux density, forces and torques have been performed by the program Ansys Maxwell. An object shown in Fig. 6 has been taken for the calculations. A circle made of metal sheet is turning round on its own rotary axis. At the top there is the sensor based on induction linear motor. All details of the sensor are explained in Fig. 3. In real conditions there is no ring of steel sheet, but simply a produced steel sheet. The simplification applied in the model is the easiest way to describe this kind of object in the program. In Fig. 7 in the middle of the steel sheet there is an air gap which symbolizes the defect of material. The air gap inside of material is moving with the steel sheet along the sensor $[3,4]$.

$\mathrm{AC}$ sensor works as a linear induction motor whose raceway moves the metal sheet. In addition, the metal sheet as a ferromagnetic material induces eddy currents effect. It is the source of forces acting on the sensor. By measuring the force, torque, and eddy current effect acting on the sensor we can identify disturbances in the magnetic field resulting from product defects.

\section{Computing methodology}

All simulations were performed with the Ansys Maxwell program. This piece of software uses the Maxwell Stress Tensors (MST) methodology [5,6,7]. Zero boundary conditions have been assigned around the machine. The area around the motor has been divided into about 300.000 triangular elements. The elements are of different dimensions, depending on the importance of the considered area. The sensitive space around the air gap, the sharp shapes around the poles, was analysed with finer mesh. The software performing the simulation is capable of modelling both dynamic and static elements. The motor was divided into the stator with 15 winding poles as the static part of the motor, and the rotor, raceway made of metal sheet as the dynamic element. The shape of the air gap between the stator and the rotor is very important [8]. The rotor axis is placed perpendicularly to the surface of screen. Considering the formulas for computing force:

$$
F=\int\left[\frac{1}{\mu_{0}} B(B \cdot n)-\frac{1}{2 \mu_{0}} B^{2} \cdot n\right] d C
$$

and torque

$$
T=r \cdot F
$$

Values in formulas (2) and (3) are as follows:

$\mathrm{B}$ - momentary value of induction in the air gap B [T]

$\mathrm{N}$ - unit vector, perpendicular to the surface of rotor,

$\mu 0$ - magnetic permeability of vacuum $\mu 0=4 \pi \cdot 107$ $[\mathrm{H} / \mathrm{m}]$.

Value of torque is calculated from the formula (3) taking the radius of rotor as $r[\mathrm{~m}]$.

It is important to assume a very big difference between magnetic permeability (minimum 1/1000) in those two analysed phases (air and iron). In the calculations the normal part of flux vectors in the air gap, and big difference of magnetic permeability between the air and electromagnetic steel were assumed. The presence of the air gap in the analysed region fulfils these conditions.

\section{Computational results}

The sensor system along with the sheet metal was modelled in the Ansys Maxwell program. Linear motor is powered according to the electrical diagram shown in Fig. 8 .

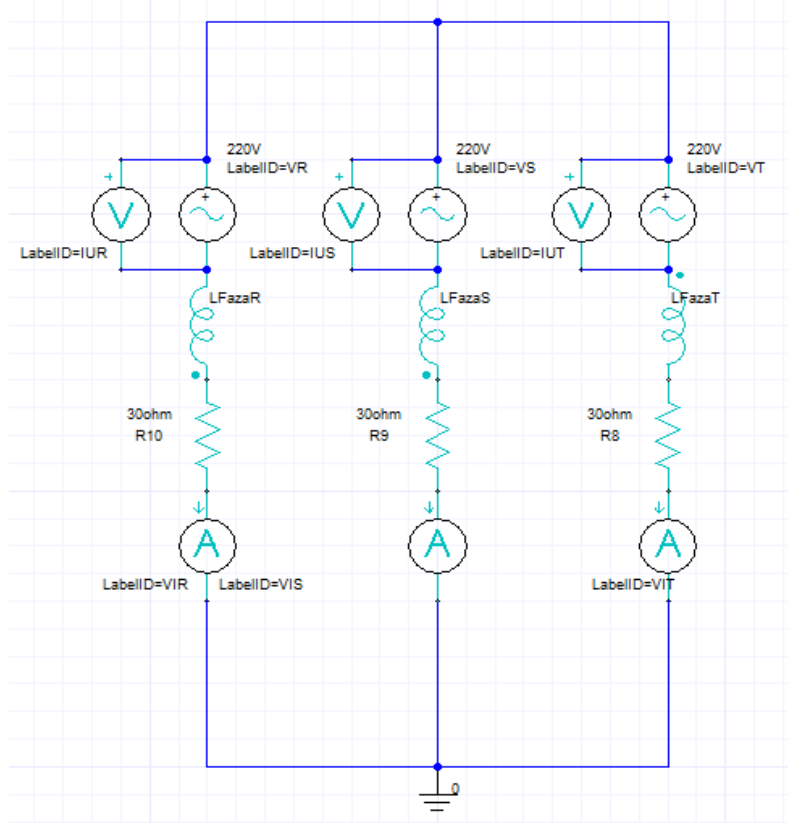

Fig. 8. Electric scheme for supplying linear induction motor.

The diagram shows three voltage sources. Sinusoidal voltage phase of each of the three sources is shifted by $120^{\circ}$ comparing to the voltage of the adjacent phases. A voltmeter was connected at each phase for voltage control. Coils of the linear motor are serially connected 
to the power source. It should be noted that, according to the scheme of the windings of the linear motor shown in Fig. 5, the coil of the phase $\mathrm{T}$ is connected in the reverse direction to the phases $\mathrm{R}$ and $\mathrm{S}$. The change of phase is necessary to correlate the direction of the moving sheet and the moving field generated by the induction motor. Serial-connected resistances limit the coil current. The ammeter connected at the end of each phase circuit controls the current connected to the linear motor. In the simulation the source of AC power at $50 \mathrm{~Hz}$ and $220 \mathrm{~V}$ was used. Simulation results are shown in the figures below.

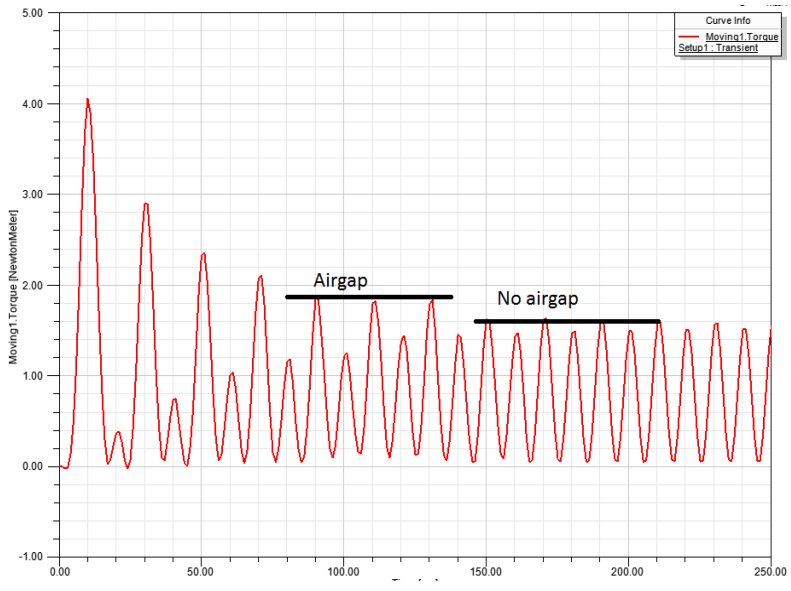

Fig. 9. Dynamic torque waveform in dependence on time.

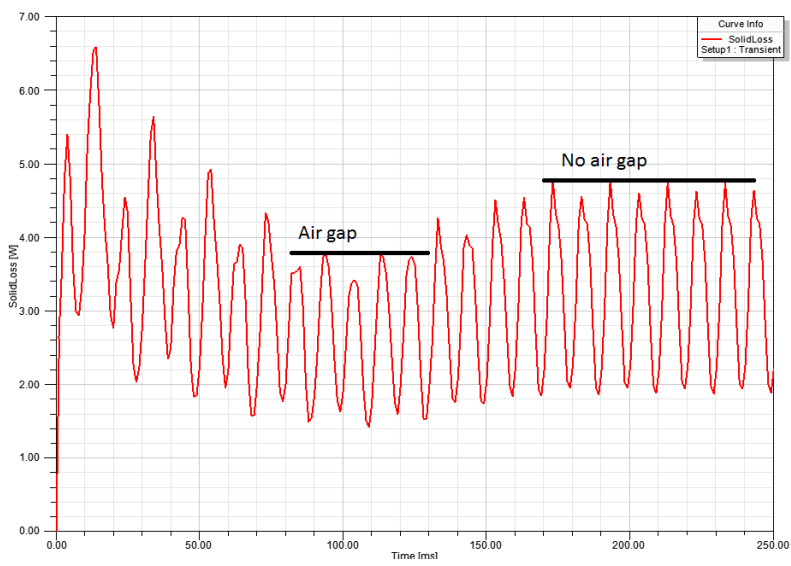

Fig. 10. Dynamic solid loss in dependence on time.

Computer simulation was made assuming the sheet thickness equal to $2 \mathrm{~mm}$ and the air gap of $0.2 \mathrm{~mm}$. The fixed state of the system was considered, which in the case shown in Fig. 9 appeared after about $80 \mathrm{~ms}$ from the time $\mathrm{t}=0$. The segment marked "air gap" is faulty, the further part of the "no air gap" is a material without defects. A comparison of the numerical results of torque over time is presented in Table 1.

Table 1. Comparison of the moving torque values with and without air gap.

\begin{tabular}{|c|c|}
\hline Air-gap 0.2 mm & Moving Torque \\
\hline With air gap [Nm] & 1.82 \\
\hline Without air gap [Nm] & 1.6 \\
\hline Difference [Nm] & 0.22 \\
\hline
\end{tabular}

Additional simulation of losses occurring in the produced sheet is also highly interesting. Due to the fact that losses from the linear motor, affecting the sheet are active, they can be distinguished by simple measurements. The losses (Eddy Current) show significant differences in size. In Fig. 10 the pass of material with and without air gap near the sensor was shown.

The simulation results presented in the time chart show a significant difference. The gap appearing in the linear motor decreases the loss by more than $20 \%$. Comparison of the results of the simulation for changes in the solid losses occurring in the test sheet is presented in Table 2.

Table 2. Value comparison for solid loss with and without air gap.

\begin{tabular}{|c|c|}
\hline Air-gap 0.2 mm & Solid loss \\
\hline With air gap [W] & 3.8 \\
\hline Without air gap [W] & 4.8 \\
\hline Difference [Nm] & 0.22 \\
\hline
\end{tabular}

\section{Conclusion}

The results of the simulation show that the construction of a sensor based on the design of an inductive linear motor for testing material quality in continuous production processes is possible. By measuring the change of torque (or thrust force) of a linear motor and changing the quality of the treadmill it is possible to generate an output signal at low air gap thicknesses. For the simulation presented in this paper, the change of torque acting on the motor at an air gap of $0.2 \mathrm{~mm}$ represents $12 \%$ of the generated torque in the steady state. It generates a signal informing the operator about the change in the quality of the material produced.

Increasing the torque acting on the sensor is explained in the second graph. It shows that in the area of the delaminated material the active loss in treadmill material is decreased. This is natural because of the reduction of eddy current losses in layered materials. Both increased torque (strength) and reduced eddy current losses can be easily measured. The noise signal given to the electronic memory system can easily be used for the distribution of the produced material. After distribution, you can verify the quality of the highlighted material to determine its suitability.

Qualitative study of materials is a broad field that uses a variety of methods. Penetration testing is a nondestructive method which employs Barkhausen effect, radiometry, $\mathrm{x}$-ray, echolocation, enables material testing resulting in higher quality of products and better performance of production machinery. This article shows the possible application of the theory of electrical machines for the construction of sensors and their use in production processes, and for the diagnostics of ferro and diamagnetic materials. The work aims to inspire designers to use existing designs in material diagnostics.

The authors intend to conduct further research by examining the effects of linear engine parameters, ferromagnetic thickness, and air gap on accuracy of 
measurements, thanks to which better sensor parameters of this type can be found.

\section{References}

1. R. Zimroz, R. Błażej, P. Stefaniak, A. Wyłomańska, J. Obuchowski, M. Hardygóra, Fundamental Problems of Conveyor Transport 21(2), 99-109 (2014)

2. T. Machula, Opracowanie metody ciagtej analizy stanu technicznego taśm przenośnikowych z linkami stalowymi, Doctoral dissertation (AGH Kraków, 2011)
3. J. Jonak, J. Gajewski, Maintenance and Reliability 4, 74-78 (2006)

4. T. Piech, Badania magnetyczne, Wykorzystanie efektu Barkhausena (Biuro Gamma, Warszawa, 2003)

5. Maxwell 15.0 Application User's Guide

6. Ansys 14.0 Application User's Guide

7. WorkBench 14 Application User's Guide

8. M. Lis, Modelowanie matematyczne procesów nieustalonych $w$ elektrycznych układach napędowych o złożonej transmisji ruchu (Wydawnictwo Politechniki Częstochowskiej, Częstochowa, 2013) 